\title{
Bayesian Information in an Experiment and the Fisher Information Distance
}

\author{
Stephen G. Walker \\ Department of Mathematics, University of Texas at Austin, Texas, U. S. A. (email: \\ S.G.Walker@math.utexas.edu)
}

\begin{abstract}
There are two forms of Fisher information; for the parameter of a model and for the information in a density model. These two forms are shown to be fundamentally connected through a measure of gain in information from a Bayesian experiment.
\end{abstract}

Keywords: Shannon entropy; information; Bayesian design.

\section{Introduction}

For the statistical model $f(x \mid \theta)$, with $x \in \mathbb{R}$ and $\theta \in \Theta \subset \mathbb{R}$, we assume that $f(x \mid \theta)$ is differentiable with respect to both $x$ and $\theta$. To start we have $\theta$ as a 1 dimensional parameter, which is extended to higher dimensions later in the paper. The amount of information about $\theta$ in a single observation experiment is given by the Fisher information;

$$
I(\theta)=\int\left(\frac{\partial}{\partial \theta} \log f(x \mid \theta)\right)^{2} f(x \mid \theta) \mathrm{d} x .
$$

It has a number of applications, most notably in large sample asymptotic studies; see, for example, [8].

On the other hand, a well known measure of the information carried by a density function is the Shannon entropy, which in the continuous case is often referred to as the differential Shannon entropy. This is defined as

$$
H(Y)=-\int f_{Y}(y) \log f_{Y}(y) \mathrm{d} y
$$


where the random variable $Y$ has density function $f_{Y}$. See [14]. This was originally defined in the discrete case; i.e. $Y$ is a discrete random variable. Some of the key properties, such as positivity, are lost in the differential case.

Nevertheless, the Shannon entropy was adopted as a measure of the information in a prior and posterior distribution; see [9]. To elaborate, suppose $p(\theta)$ denotes a prior distribution for model $f(x \mid \theta)$ and $p(\theta \mid x)$ is the posterior distribution. The idea is that the gain in information by observing outcome $X$ is given by

$$
\Delta(X)=H(\Theta \mid X)-H(\Theta)
$$

where

$H(\Theta)=-\int p(\theta) \log p(\theta) \mathrm{d} \theta \quad$ and $\quad H(\Theta \mid X=x)=-\int p(\theta \mid x) \log p(\theta \mid x) \mathrm{d} \theta$.

The expected gain in information by the experiment is therefore given by $\Delta=\int \Delta(x) m(x) \mathrm{d} x$ where $m(x)=\int f(x \mid \theta) p(\theta) \mathrm{d} \theta$. One can easily find simplified forms for $\Delta$, which can also be represented as

$$
\Delta=\int D(p(\cdot \mid x), p(\cdot)) m(x) \mathrm{d} x
$$

where $D$ denotes the Kullback-Leibler divergence;

$$
D\left(p_{1}, p_{2}\right)=\int p_{1}(x) \log \frac{p_{1}(x)}{p_{2}(x)} \mathrm{d} x .
$$

Applications in the Bayesian design of experiments can be found in [13].

If $I(\theta)$ represents the information about $\theta$ in a single observation experiment and $p(\theta)$ is the prior, then we ask why the expected information in the experiment is not simply

$$
\widetilde{\Delta}=\int I(\theta) p(\theta) \mathrm{d} \theta,
$$

rather than $\Delta$. We will discuss this, showing that (4) arises by using the Fisher information distance, $F\left(p_{1}, p_{2}\right)$ (to be defined later), i.e.

$$
\widetilde{\Delta}=\int F(p(\cdot \mid x), p(\cdot)) m(x) \mathrm{d} x,
$$

rather than the Kullback-Leibler divergence, in section 3. However, for now, we note that $\int I(\theta) p(\theta) \mathrm{d} \theta$ does appear in a Bayesian type Cramér-Rao bound; see [4]. 
In section 2 we show that an alternative recognized measure of information of a density, $J(Y)$, also known as the Fisher information, is such that if the gain in information given observation $X$ is

$$
\Delta^{*}(X)=J(\Theta \mid X)-J(\Theta)
$$

then the expected gain,

$$
\Delta^{*}=\int \Delta^{*}(x) m(x) \mathrm{d} x
$$

coincides with $\widetilde{\Delta}$.

\section{Fisher information for a density function}

Widely used in the functional analysis community, the Fisher information for a density $f_{Y}$ is given by

$$
J(Y)=\int \frac{f_{Y}^{\prime}(y)^{2}}{f_{Y}(y)} \mathrm{d} y .
$$

See, for example, [2]. This can also be represented as

$$
J(Y)=\int f_{Y}(y)\left(\frac{\partial}{\partial y} \log f_{Y}(y)\right)^{2} \mathrm{~d} y
$$

and of course $J(Y)$ will coincide with (1) when $f_{Y}(y)=f(y-\theta)$; i.e. a location model.

Now (5) is not so well known in the statistics literature; though there are some references. For example, [3] used it to prove the central limit theorem via a Cramér-Rao inequality, and elaborating on this paper, [7], also use (5), as well as the Fisher information distance, to be defined later in this paper. As well, [11] discuss properties of (5) as a direct comparison with the properties of (1).

Theorem 1. It is that $\Delta^{*} \equiv \widetilde{\Delta}$.

Proof. Now

$$
J(\Theta \mid X=x)=\int \frac{\left(\frac{\partial}{\partial \theta} p(\theta \mid x)\right)^{2}}{p(\theta \mid x)} \mathrm{d} \theta
$$


which is given by

$$
\frac{1}{m(x)} \int \frac{\left(p^{\prime}(\theta) f(x \mid \theta)+p(\theta) \frac{\partial}{\partial \theta} f(x \mid \theta)\right)^{2}}{p(\theta) f(x \mid \theta)} \mathrm{d} \theta .
$$

Taking expectations with respect to $m(x)$, and noting that

$$
\iint p^{\prime}(\theta) \frac{\partial}{\partial \theta} f(x \mid \theta) \mathrm{d} x \mathrm{~d} \theta=0,
$$

allowing the usual interchanging of integration and differentiation ([1] and [6], p. 518) ${ }^{1}$ we see that

$$
\int[J(\Theta \mid x)-J(\Theta)] m(x) \mathrm{d} x=\iint \frac{\left(\frac{\partial}{\partial \theta} f(x \mid \theta)\right)^{2}}{f(x \mid \theta)} \mathrm{d} x p(\theta) \mathrm{d} \theta .
$$

The right-hand-side is then seen to be

$$
\iint\left(\frac{\partial}{\partial \theta} \log f(x \mid \theta)\right)^{2} f(x \mid \theta) \mathrm{d} x p(\theta) \mathrm{d} \theta
$$

as required.

Now $\widetilde{\Delta}$ can also be represented as the expected relative Fisher information distance between the prior and posterior. This distance between densities $p_{1}$ and $p_{2}$ is given by

$$
F\left(p_{1}, p_{2}\right)=\int p_{1}(y)\left(\frac{p_{1}^{\prime}(y)}{p_{1}(y)}-\frac{p_{2}^{\prime}(y)}{p_{2}(y)}\right)^{2} \mathrm{~d} y,
$$

and is well known in transport theory.

Lemma 2. It is that

$$
\widetilde{\Delta}=\int m(x) F(p(\cdot \mid x), p(\cdot)) \mathrm{d} x .
$$

\footnotetext{
${ }^{1}$ Sufficient conditons are given as $\partial / \partial \theta f(x \mid \theta)$ exists for all $x$, and there exists a Lebesgue integrable function $g(x)$ such that $\left|\left(f\left(x \mid \theta_{1}\right)-f\left(x \mid \theta_{2}\right)\right) /\left(\theta_{1}-\theta_{2}\right)\right| \leq g(x)$ for all $x$ and $\theta_{1} \neq \theta_{2}$
} 
Proof. This is straightforward to prove and relies on the fact that

$$
\iint m(x) \frac{\partial / \partial \theta p(\theta \mid x) p^{\prime}(\theta)}{p(\theta)} \mathrm{d} \theta \mathrm{d} x=\int \frac{p^{\prime}(\theta)^{2}}{p(\theta)} \mathrm{d} \theta .
$$

We can consider both the expected Kullback-Leibler divergence or the expected relative Fisher information distance as a measure of the difference between prior and posterior; interpreted as the expected gain in information in a Bayesian experiment. The attractive feature of using the relative Fisher information distance is the result of the expected distance being the expected Fisher information.

The multivariate versions of $\Delta^{*}$ and $\widetilde{\Delta}$, when $\theta \in \Theta \subset \mathbb{R}^{d}$, also match when the (Fisher) information in $\theta$ is given by $I(\theta)=\operatorname{trace} \mathcal{I}(\theta)$, where $\mathcal{I}(\theta)$ is the $d \times d$ Fisher information matrix; i.e.

$$
\mathcal{I}_{i j}(\theta)=\int\left[\left(\frac{\partial}{\partial \theta_{i}} \log f(x \mid \theta)\right)\left(\frac{\partial}{\partial \theta_{j}} \log f(x \mid \theta)\right)\right] f(x \mid \theta) \mathrm{d} x .
$$

To derive this, we start with

$$
J(\Theta \mid X)=\int \frac{|\nabla f(x \mid \theta)|^{2}}{f(x \mid \theta)} \mathrm{d} \theta
$$

which is equal to

$$
\frac{1}{m(x)} \int \frac{\sum_{j=1}^{d}\left(p(\theta) \frac{\partial}{\partial \theta_{j}} f(x \mid \theta)+f(x \mid \theta) \frac{\partial}{\partial \theta_{j}} p(\theta)\right)^{2}}{p(\theta) f(x \mid \theta)} \mathrm{d} \theta
$$

Taking expectations with respect to $m(x)$, noting that the cross terms are 0 ; i.e.

$$
\iint \frac{\partial}{\partial \theta_{j}} p(\theta) \frac{\partial}{\partial \theta_{j}} f(x \mid \theta) \mathrm{d} x \mathrm{~d} \theta=0
$$

we see that

$$
\int[J(\Theta \mid x)-J(\Theta)] m(x) \mathrm{d} x=\iint \sum_{j=1}^{d}\left(\frac{\partial}{\partial \theta_{j}} \log f(x \mid \theta)\right)^{2} f(x \mid \theta) p(\theta) \mathrm{d} x \mathrm{~d} \theta
$$

as required. 


\section{Bayesian design}

The connection between the two forms of Fisher information suggests that

$$
\widetilde{\Delta}=\operatorname{trace} \int \mathcal{I}(\theta) p(\theta) \mathrm{d} \theta
$$

represents the gain in information in a Bayesian experiment with model $f(x \mid \theta)$ and prior $p(\theta)$.

Such results are associated with the design of a Bayesian experiment where the model is $f(x \mid \theta, \xi)$ and the idea is to select the $\xi$ which maximizes the gain in information about $\theta$. For us, based on $\widetilde{\Delta}$, we would choose the $\xi$ which maximizes

$$
\Delta_{T}=\operatorname{trace} \int \mathcal{I}_{\xi}(\theta) p(\theta) \mathrm{d} \theta=\int m(x) F(p(\cdot \mid x, \xi), p(\cdot)) \mathrm{d} x,
$$

where $\mathcal{I}_{\xi}(\theta)$ is obtained from the model $f(x \mid \theta, \xi)$, and in the multivariate case

$$
F\left(p_{1}, p_{2}\right)=\int p_{1}\left|\frac{\nabla p_{1}}{p_{1}}-\frac{\nabla p_{2}}{p_{2}}\right|^{2}
$$

Now the most popular approach to Bayesian design is based on the expected Kullback-Leibler divergence between prior and posterior; i.e. maximize

$$
\Delta_{B}=\int m(x) D(p(\cdot \mid x, \xi), p(\cdot)) \mathrm{d} x .
$$

For a recent application, see [12].

On the other hand, selecting $\xi$ via expected gains in information using $\mathcal{I}_{\xi}(\theta)$ are regarded as pseudo-Bayesian. Two popular ideas are the pseudoBayesian D-design;

$$
\Delta_{D}=\int \log \operatorname{det} \mathcal{I}_{\xi}(\theta) p(\theta) \mathrm{d} \theta
$$

see [5], for example, and the pseudo-Bayesian A-design;

$$
\Delta_{A}=\int \operatorname{trace} \mathcal{I}_{\xi}(\theta)^{-1} p(\theta) \mathrm{d} \theta
$$

where here one minimizes $\Delta_{A}$. See [10], for example.

However, there is nothing pseudo-Bayesian about (7), which would be known as a pseudo-Bayesian T-design, given the derivation involves simply 
replacing the Kullback-Leibler divergence with the relative Fisher information distance.

Here we present a simple illustration; though there are also numerous cases in the literature. Suppose $Y$ is Poisson with parameter $e^{\xi \theta}$ where $\xi>0$ is a design point, to be chosen. It is easy to obtain the Fisher information for $\theta$ as

$$
I_{\xi}(\theta)=\xi^{2} e^{-\xi \theta} .
$$

Hence, we would select $\xi$ by maximizing

$$
\Delta_{T}=\xi^{2} \int e^{-\xi \theta} p(\theta) \mathrm{d} \theta .
$$

So if $p(\theta)$ were gamma with parameters $(a, b)$, with $a>2$, then

$$
\Delta_{T}=\frac{\xi^{2}}{(b+\xi)^{a}}
$$

which is maximized for $a>2$ by $\xi=2 b /(a-2)$.

\section{Discussion}

There are two notions of Fisher information; i.e. (1), which provides information about the parameter of a model, and (5), which provides information about the density itself. When associated with the gain in information in a Bayesian experiment, these two complement each other perfectly, via theorem 2.1. In fact, whereas [9] uses the expected Kullback-Leibler divergence between prior and posterior, we propose the expected relative Fisher information distance between prior and posterior, due to its connection with the expected Fisher information about $\theta$.

If (8) is regarded as fully Bayes, then so must (7), rather than as a pseudoBayesian approach, due to its derivation as a measure of distance between prior and posterior.

\section{Acknowledgments.}

I am grateful for the comments and suggestions of two referees, and from an Associate Editor. Research partially supported by NSF grant DMS 1506879 . 


\section{References}

[1] T. M. Apostol, Mathematical Analysis, (1957), Tokyo: AddisonWesley.

[2] S. G. Bobkov, N. Gozlan, C. Roberto and P. M. Samson, On bounds on the deficit in the logarithmic Sobolev ineuquality, J. Funct. Analysis 267 (2014), 4110-4138.

[3] L. D. Brown, A proof of the central limit theorem motivated by the Cramer-Rao inequality, Statistics \& Probability: Essays in Honor of C.R.Rao. (Kallianpur et al., eds). (1982), 141-148.

[4] R. D. Gill And B. Y. Levit, Applications of the van Trees inequality: a Bayesian Cramér-Rao bound, Bernoulli 1 (1995), 59-79.

[5] C. M. Gotwalt, B. A. Jones And D. M. Steinberg, Fast computation of designs robust to parameter uncertainty for non-linear settings, Technometrics 51 (2009), 88-95.

[6] J. H. Hubbard and B. B. Hubbard, Vector Calculus, Linear Algebra, and Differential Forms, 2nd Edition, Prentice Hall, NJ. (2002).

[7] O. Johnson And A. Barron, Fisher information inequalities and the central limit theorem, Probability Theory and Related Fields 129 (2004), 391-409.

[8] E. L. Lehmann And G. Casella, Theory of Point Estimation (2nd ed.). (1998), Springer.

[9] D. V. Lindley, On the measure of information provided by an experiment, Ann. Math. Statist. 27 (1956), 986-1005.

[10] N. K. Mandal, M. Pal and M. L. Aggarwal, Pseudo-Bayesian A-optimal designs for estimating the point of maximum in componentamount Darroch-Waller mixture model, Statistics and Probability Letters 82 (2012), 1088-1094.

[11] T. Papaionnnou and K. Ferentinos, On two forms of Fisher's measure of information, Communications in Statistics - Theory and Methods 34 (2005), 1461-1470. 
[12] E. G. Ryan, C. C. Drovandi And A. N. Pettitt, Fully Bayesian experimental design for pharmacokinetic studies, Entropy 17 (2015), 1063-1089.

[13] P. Sebastiani And H. P. Wynn, Bayesian experimental design and Shannon information, In 1997 Proceedings of the Section on Bayesian Statistical Science (1997), 176-181.

[14] C. E. Shannon, A mathematical theory of communication, Bell Labs Technical Journal 27 (1948), 379-423. 